\title{
Modification in the Kerberos Assisted Authentication in Mobile Ad-Hoc Networks to Prevent Black Hole Attack
}

\author{
Manpreet Kaur \\ M Tech Scholar(C.S.E) \\ Amritsar College of Engineering \\ \& Technology \\ Amritsar, India.
}

\author{
Tanu Preet Singh \\ H.O.D(C.S.E) \\ Amritsar College of Engineering \\ \& Tchnology \\ Amritsar,India.
}

\author{
Barjinder Singh \\ Lecturer(C.S.E) \\ Lovely Professional University \\ Jalandhar, India.
}

\begin{abstract}
MANET is the self-configuring type of network in which the mobile nodes can leave or join the network when they want. MANET is decentralized type of network, no central controller is present. Due to their unique features mobile ad hoc networks can be deployed anywhere round the clock. This posed the remedial venture to large number of attacks like replay attack, fabrication, eavesdropping etc Kaman provides secure solution to the problem of secure channel establishment, secure exchange of session keys and prevention of nodes identity forgery. In this paper, we reviewed the Kaman; Kerberos assisted Authentication in Mobile Ad hoc Network and added the concept of timers in KAMAN to solve the problem of black hole attack that aroused when Kaman protocol is embedded into large network AODV, on-demand routing protocol had been used to select secure shortest path between the nodes.
\end{abstract}

\section{Keywords}

Black hole, Mutual Authentication, Secure server, MANET, KAMAN.

\section{INTRODUCTION}

Mobile Ad Hoc Network consists of wireless mobile nodes where each node acts as a router that forwards the packets from one node to another node. In MANET nodes are free to move and hence topology of MANET is very dynamic. Such characteristics allow an ad hoc network to be established on the fly with built in fault tolerance and unconstrained connectivity. This makes routing in such networks more challenging, especially when certain Quality of Service requirements are to be guaranteed during the routing. There is no fixed infrastructure in the MANET therefore each node must cooperate for forwarding the packet from source node to destination node. To form such a cooperative and selfconfigurable environment, every mobile host must be willing to relay messages from other hosts to their ultimate destinations. In such a network, it may be necessary for one mobile host to enlist the other hosts in forwarding a packet to its destination, due to the limited range of each mobile host's wireless transmissions. This type of wireless network is known as mobile ad hoc network. If there are only two hosts in the mobile ad hoc network then we cannot see the real routing process involved in the MANET. In many ad hoc networks, though, two hosts that want to communicate may not be within wireless transmission range of each other, but could communicate if other hosts between them also participating in the ad hoc network are willing to forward

packets for them. To establish efficient direct or in-direct communication link between the independent nodes of ad-hoc network, a trust relationship must be maintained between every node in ad hoc network. An efficient mechanism to maintain trust relationship between every node in ad hoc networks is mutual authentication. Before communicating, with other nodes in the network, every node must be mutually authenticated. This has prevented many types of active and passive attacks.

Mutual Authentication can be accomplished in two ways:-

\section{Direct authentication. \\ Indirect authentication.}

In direct authentication, both parties use symmetric and asymmetric authentication algorithms for authentication. Whereas, in direct authentication incorporated the use of third party. Authentication scheme proposed in the Kerberos authentication is a hybrid type of authentication scheme. Kerberos scheme is the combination of indirect and direct authentication.

\section{LITERATURE REVIEW}

Asad Amir Pirzada and Chris Kaman, Kerberos assisted Authentication in Mobile Ad-hoc Networks, a new pure managed authentication service for mobile ad-hoc networks. Kaman is based on the time-tested and widely deployed Kerberos protocol, and provides secure extensions to support the more challenging demands of ad-hoc networks. Kaman migrates a number of features from the traditional, wired Kerberos environments to the ad-hoc environment, including the prevention of node identity forgery, the detection of replay attacks, establishment of secure channels, mutual endpoint authentication, and the secure distribution of provisional session keys amongst replicated servers.[1] Kerberos tickets used in KAMAN authentication scheme can be captured over the network are prone to replay attacks. Modification in KAMAN protocol can increase authorization. All of contents are encapsulated in an encrypted packet. So the replay attacks become impossible. To prevent reply attack they add session parameter in the Ticket grand message [2]. Semih Dokurer and Y. M. Erten and Can Erkin Acar investigated the effects of black hole attack on the network performance. A wireless ad-hoc network is a temporary network set up by wireless nodes usually moving randomly and communicating without a network infrastructure. Due to security vulnerabilities of the routing protocols, however, wireless ad-hoc networks may be unprotected against attacks by the malicious nodes [3]. Failures may cause data packets to be silently dropped inside the network without triggering any alarms or responses (e.g., the failure is not routed around). So-called "silent failures" or "black holes" represent a critical threat to today's rapidly evolving networks. In this paper, we present a simple and effective method to detect and diagnose such silent failures. Our method uses active measurement between edge routers to raise alarms whenever end to end connectivity is disrupted, regardless of the cause. These alarms feed localization agents that employ spatial correlation techniques to isolate the rootcause of failure [4]. Black hole attacks occur when an adversary captures and re-programs a set of nodes in the 
network to block/drop the packets they receive/generate instead of forwarding them towards the base station. As a result any information that enters the black hole region is captured. Black hole attacks are easy to constitute and they are capable of undermining network effectiveness by partitioning the network, such that important event information do not reach the base stations. In this paper, they may even end up making black hole attacks more effective. We propose an efficient technique that uses multiple base stations deployed in the network to counter the impact of black holes on the data transmission [5].

\section{KERBEROS ASSISTED AUTHENTICATION PROTOCOLIN MOBILE AD HOC NETWORKS}

Kerberos assisted authentication protocol is the extension of traditional Kerberos authentication protocol. To prevent various types of active and passive attacks in wireless ad-hoc network every node in the ad-hoc network should be mutually authenticated. Kerberos assisted authentication protocol eliminates various disadvantages of traditional Kerberos authentication protocol. As much numbers of message is needed for successful authentication which leads to degrade the battery performance of the mobile devices .In KAMAN only two parties are involved while authentication but in traditional Kerberos protocol third party had involve while authentication. The third, main disadvantage of traditional Kerberos authentication protocol is the assumptions that we assume while implementing the protocol in the actual environment when the environment in which Kerberos protocol is embedded change Kerberos protocols performance factors degrades. We assume while implementing the protocol in the actual environment when the environment in which Kerberos protocol is embedded change Kerberos protocols performance factors degrades.

Three assumptions are taken into consideration while implementing Kaman these assumptions are:

- Hashed passwords of all users are stored in the server, all users have passwords and they are only known to them.

- All servers are mutually authenticated and share a secret key.

- All servers shared secrete key .Repository are encrypted with the secrete key when replication takes place.

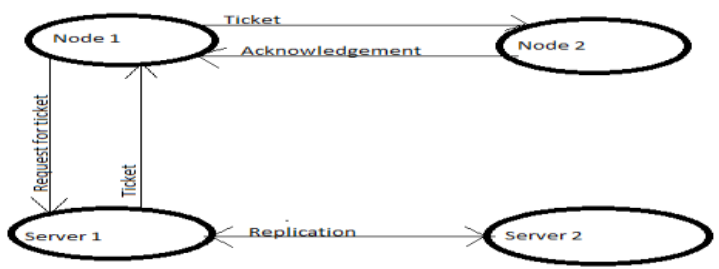

Figure 1: Operations of kaman

Suppose two mobile nodes are node 1 and node 2. Server1 and server 2 are the authentication servers. When mobile node 1 wants to communicate with node 2 , Node1 and node 2 should be mutually authenticated with the authentication server. For authentication, node 1 requests for a ticket to server 1 . When node 1 has been successfully authenticated, server 1 sends ticket to node 1.Ticket contains the virtual ids of node 1 and node 2. Shared key communication between node 1 and node 2 is encrypted with shared key and tickets are encrypted with public key of node 1 . When node 1 receives ticket, it decrypts the ticket with its own private key and encrypts the same ticket with the public key of node 2 . When node 2 receives, ticket from node 1, it will decrypt that ticket with its own private key. After receiving the ticket, node 2 sends acknowledgment to node1. On receiving the acknowledgment node 1 , starts the communication with node 2 . The servers, server1 and server 2 have been replicated. In KAMAN we will assume that hashed passwords are stored on the authentication servers and each server is mutually authenticated with other server.

\section{PROPOSED WORK}

This work is about wireless ad hoc networks . The purpose of this work is to promote the secure and reliable data transmission. It has been attained by the use of Kerberos assisted authentication protocol with multipath routing ADOV protocol .Kerberos assisted authentication protocol have been used for the mutual authentication, to maintain the trust relationship between the mobile nodes and multipath routing protocol AODV for fast data transmission. Here our work is based on two methods

- Kerberos assisted authentication protocol

- Multiple routing protocol AODV

We are implementing the KAMAN model in large network and embedded AODV routing protocol with the same. The network had been set up with finite number of nodes and servers, along with defining the source and destination nodes. By using AODV routing protocol, source chooses the shortest path between source and server. Source wishes to communicate with destination. So prior to communication, there must be mutual authentication established between the two parties. For mutual authentication, source requests to its nearest server. The source sets the threshold value of timer. If source gets the ticket within threshold value, then sends the same to the destination for mutual authentication. If not, the source has to change its path, as Black hole has been triggered and it drops the ticket. So, source has to make the request to the server for ticket again. When source gets successfully authenticated to Server, Server then issues Ticket to source. If the ticket is successfully received by the source afterwards, source passes that Ticket to destination. When destination receives, the Ticket it sends the acknowledgement to source. Ticket contains the shared key which is generated by the Server. Data exchanged between source and destination is encrypted by using shared key. Server 1 and Server 2 both are mutually authenticated. The servers are self-replicating and keep on producing their replicas from time to time. In KAMAN, we have assumed that hashed passwords are stored on the authentication servers and each server is mutually authenticated with other server. 


\section{RESEARCH METHODOLOGY}

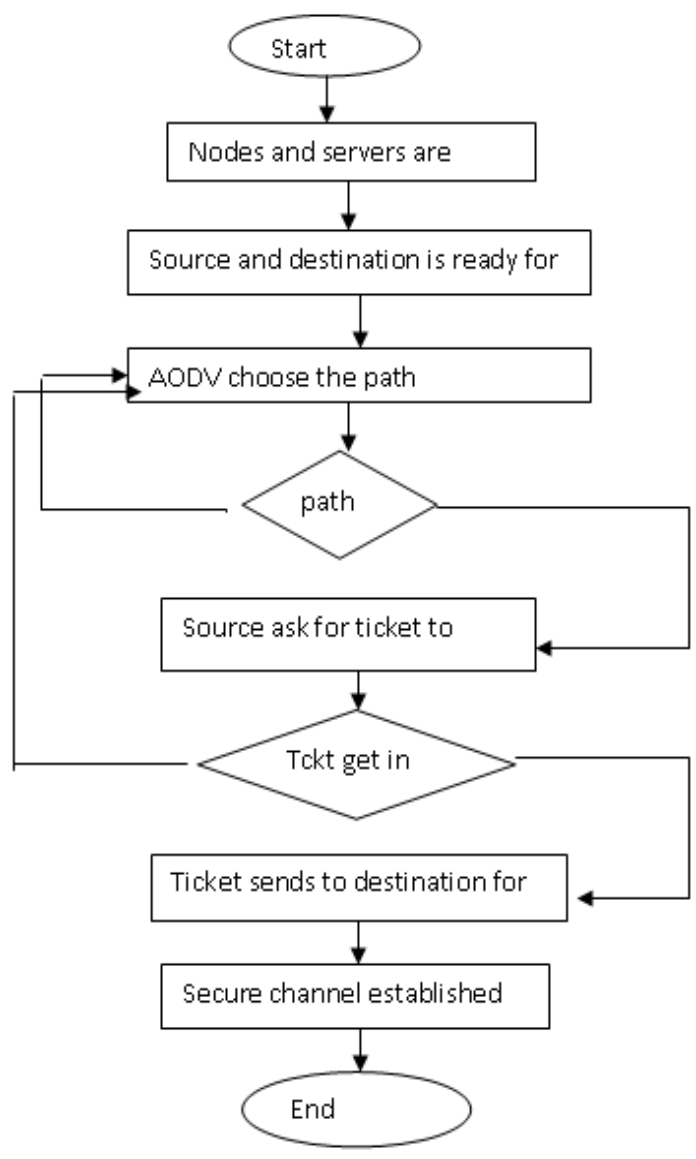

Figure 2: Flow Chart of Proposed Work

\section{SIMULATION RESULTS}

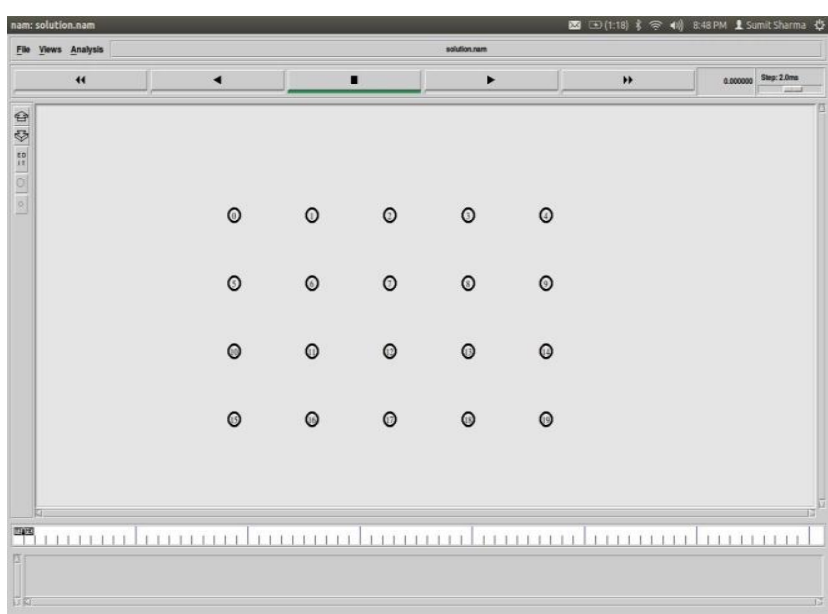

Figure 3: network deployment

The network is deployed with the finite number of mobile nodes. In the deployed network some mobile nodes become server on the basis of usage

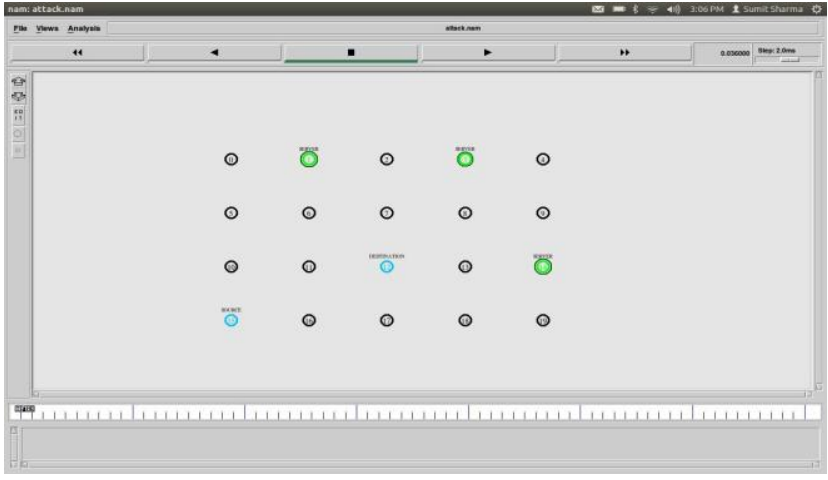

Figure 4: Server formation

The certain mobile are formed as the servers. The nodes are formed as servers on the basis of usage. The hash passwords of the mobile nodes are stored on the servers.

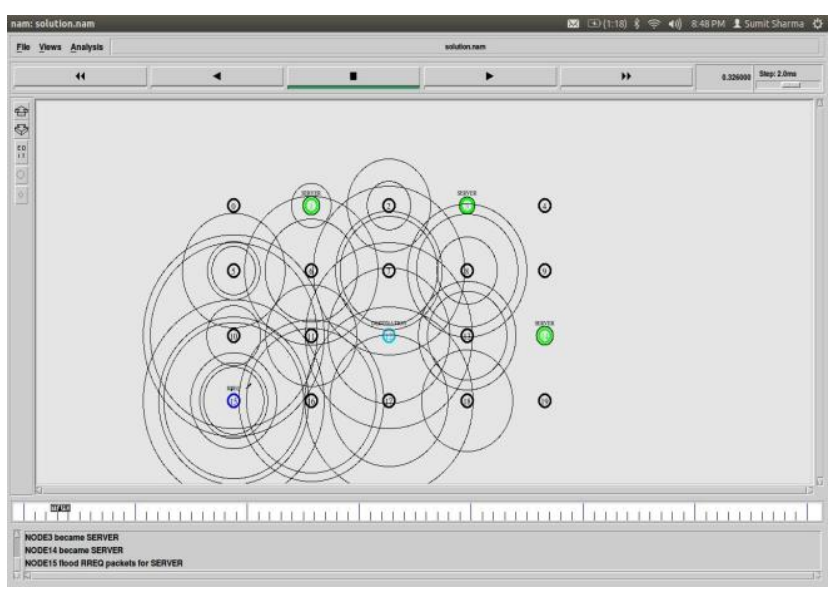

Figure 5: Route request packets flooding

The source nodes flood the route request packets in the network. The intermediate nodes which is having path to server will reply back with the route reply packets

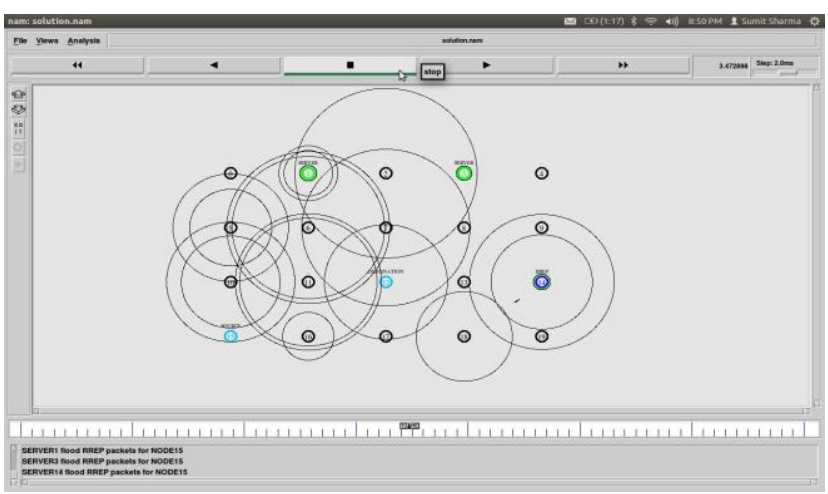

Figure 6: Best Path Selection

The source node selects the best path to destination on the basis of hop counts and sequence number. The route which is having minimum number of hop counts and higher sequence number is selected as best route 


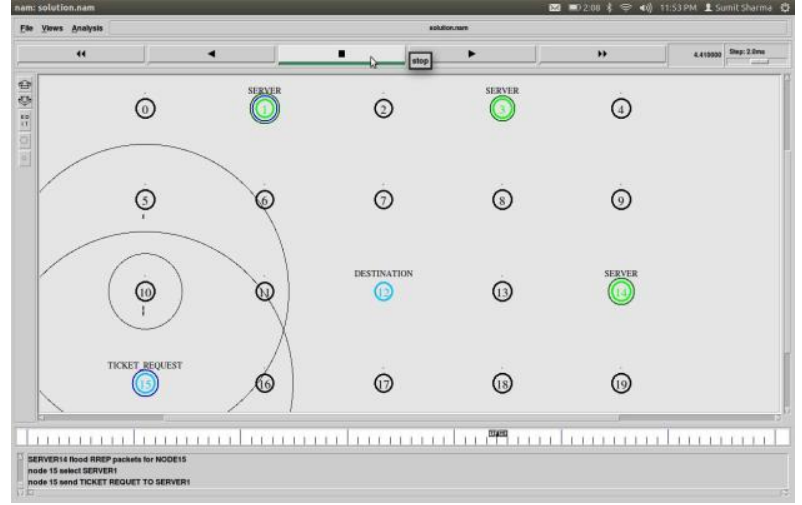

Figure 7: Request for Ticket

When the route is established between source and server, source requests for the ticket.

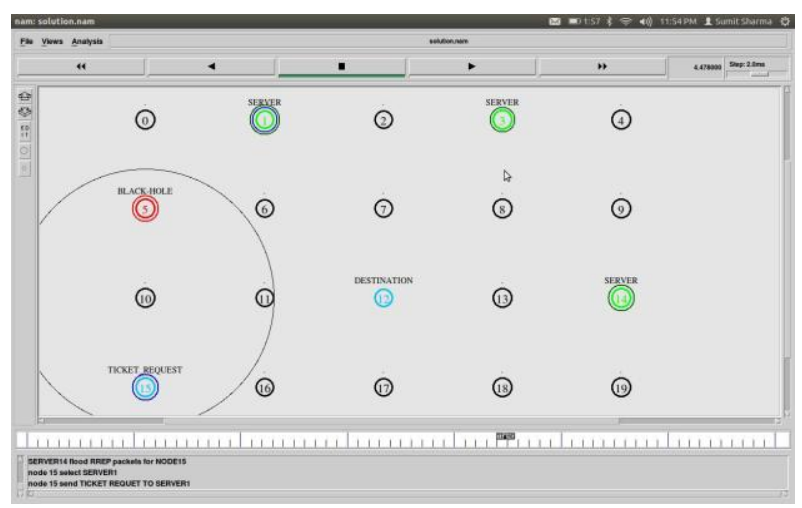

Figure 8: Black hole Problem

The source node requests for ticket to server. In the route which is between source and server in this route some malicious nodes can exists. The malicious nodes are responsible for triggering the black hole attack. The malicious node keep on dropping the packets and source will wait for ticket.

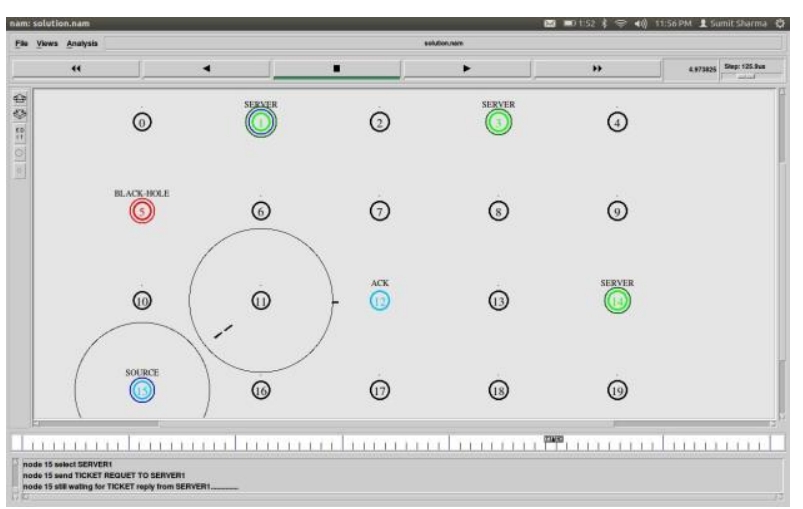

\section{Figure 9: Change of Path}

When source is not able to get ticket for threshold period of time, it changes its path and selects the second best path. Through the second path source request for the ticket

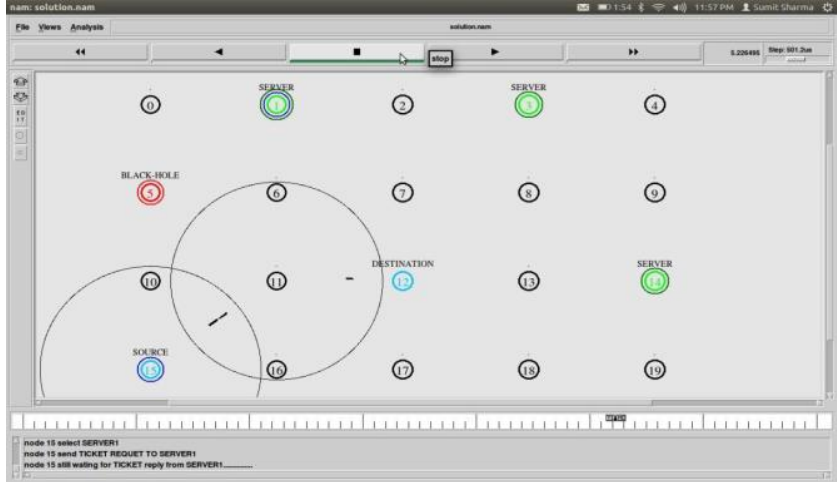

Figure 10: Communication between source and destination

When the source node gets ticket through the second best path, the communication starts between source and destination.

\section{COMPARISON GRAPHS \\ 7.1 Delay}

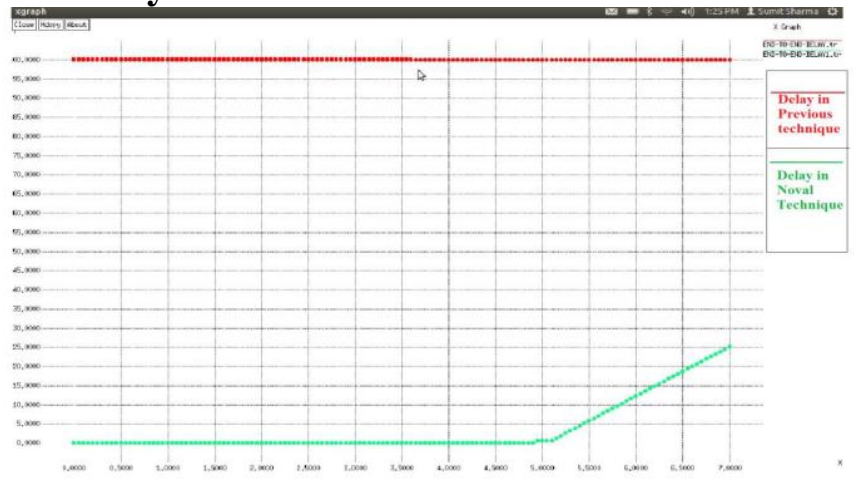

Figure 11: Comparison graphs of Delay

The y axis of the graph represents the number of packets and $\mathrm{x}$ axis represents the time. The delay in previous technique is maximum due to black hole problem. In novel approach delay will be constant for certain period of time, and then take hike to certain constant value.

\subsection{Energy}

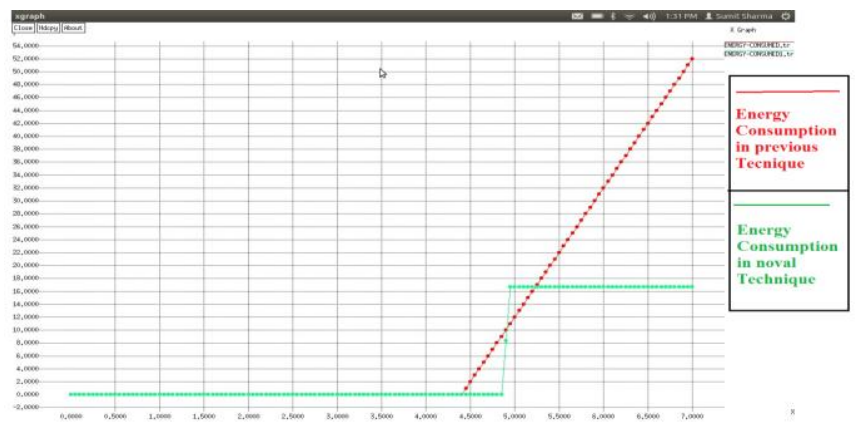

Figure 12: Comparison graphs of Energy

It can be seen from the graph that energy has been increasing with the increase in the number of packets. There has been a considerable hike in the amount of energy increased, if we compare the statistics of the previous and novel technique.

\subsection{Throughput}




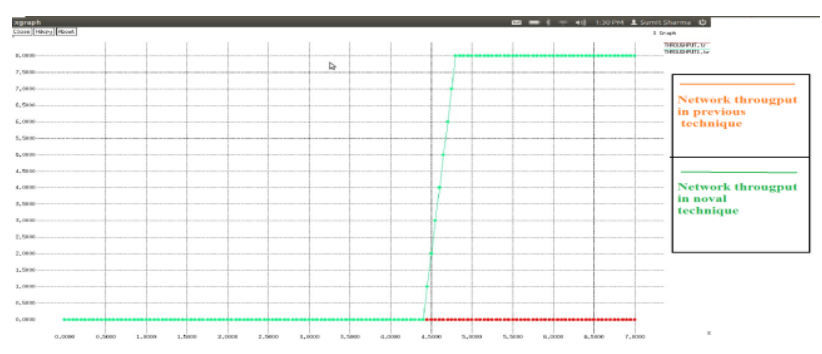

Figure 13: Throughput comparison Graph

The $y$ axis of the graph represents the number of packets and $\mathrm{x}$ axis represents the time. The throughput of network is zero due to the problem of black hole. In novel approach when black hole problem solved, network throughput take hike for constant duration, and become constant.

\section{CONCLUSIONS AND FUTURE WORK}

In this paper we conclude that when Kaman will be implemented in larger network, some routing protocol is needed for routing the packets, here we have used AODV reactive routing protocol. Which opened room for the black hole problem. This work can be extended to fix this problem and can be used for the networks with much wider domain using Kaman model.

\section{REFERENCES}

[1] Asad Amir Pirzada and Chris McDonald,"Kerberos Assisted Authentication in Mobile Ad-hoc Networks

[2] Kashif Bashir and Mohammad Khalid Khan,"Modification in Kerberos Assisted Authentication in Mobile Ad-Hoc Networks to Prevent Ticket Replay Attacks", IEEE 2012.

[3] Semih Dokurer and Y. M. Erten and Can Erkin Acar,"Performance analysis of ad-hoc networks under black hole attacks", IEEE 2007.

[4] Satyajayant Misra, Kabi Bhattarai and and Guoliang Xue,"BAMBi: Blackhole Attacks Mitigation with Multiple Base Stations in Wireless Sensor Networks",IEEE 2011.

[5] Shashidhar M S and Suresha D," Implementation of Secure Biometric Authentication Using Kerberos Protocol", 2013.

[6] Monika, Mukesh Kumar and Rahul Rishi,"Security Aspects in Mobile Ad Hoc Network (MANETs): Technical Review”, IEEE 2010.

[7] Trapti Ozha," Kerberos: An Authentication Protocol ", IEEE 2013.

[8] Ramana Rao Kompella, Jennifer Yates, Albert Greenberg, and Alex C. Snoeren,"Detection and Localization of Network Black Holes", IEEE 2007.

[9] Andreas Hafslund and Jon Andersson, Thales Norway AS (2006),"2-Level Authentication Mechanism in an Internet connected MANET", 6th Scandinavian Workshop on Wireless Ad-hoc Networks.

[10] Adebanjo Adekiigbe, ,Kamalrulnizam Abu Bakar And Ogunnusi Olumide Simeon (2011), "A Review of
Cluster-Based Congestion Control Protocols in Wireless Mesh Networks",1 IJCSI International Journal of Computer Science Issues, Vol. 8, Issue 4, No 2, July 2011 ISSN (Online): 1694-0814.

[11] C.E. Perkins, P. Bhagwat, "Highly Dynamic DestinationSequenced Distance-Vector Routing (DSDV) for Mobile Computers", SIG- COMM ${ }^{r e 9}$ Conference on Communications Architectures, Protocols and Applications, August 2008, pp. 234-244.

[12] C. Siva Ram Murthy, B. S. Manoj, 2007 "Ad Hoc Wireless Networks, Architectures and Protocols"

[13] Ching-Wen Chen, Ming-Chin Chuang, "An Efficient Authentication Scheme between MANET and WLAN Based on Mobile IPv6", International Journal of Network Security, Vol.1, No.1, PP .14-23, July 2005 (http://isrc.nchu.edu.tw/ijns/).

[14] D. Liu, P. Ning, (2008) "Establishing pairwise keys in distributed sensor networks", ACM Conference on Computer and Communications Security (CCS), October 2008.

[15] D. Liu, P. Ning, (2004) "Multi-Level u-TESLA: a broadcast authentication system for distributed sensor networks", ACM Transactions in Embedded Computing Systems (TECS), vol. 3 (4), 2004, pp. 800-836.

[16] David B. Johnson David A. Maltz Josh Broch," DSR: The Dynamic Source Routing Protocol for Multi-Hop Wireless Ad Hoc Networks.

[17] Georgios Kambourakis, Elisavet Konstantinou," Efficient Certification Path Discovery for MANET".

[18] Georgy Sklyarenko, (2009) "AODV Routing Protocol” Institute Info rmatik ,Freie University at Berlin, European Journal of Scientific research.

[19] Govind Sharma, Manish Gupta (2012)," Black Hole Detection in MANET Using AODVIRouting Protocol",

[20] International Journal of Soft Computing and Engineering (IJSCE) ISSN: 2231-2307, Volume-1, Issue-6, January 2012.

[21] Irshad Ullah,Shoaib UR Rehman,2010 "Analysis of Black Hole attack On MANETs Using different MANET Routing Protocols"

[22] Kimaya Sanzgiri Bridget Dahill Brian Neil Levine Clay Shields Elizabeth M. Belding-Royer, "A Secure Routing Protocol for Ad Hoc Networks", Dept. of Computer Science, University of California, Santa Barbara, CA 93106.

[23] Kamarularifin Abd. Jalil , Zaid Ahmad, Jamalul-Lail Ab Manan, "Mitigation of Black Hole Attacks for AODV Routing Protocol", Faculty of Computer and Mathematical Sciences, Universiti Teknologi MARA Malaysia, Shah Alam, Selangor, Malaysia.

[24] Kashif Bashir and Mohammad Khalid Khan,(2012), "Modification in Kerberos Assisted Authentication in Mobile Ad-Hoc Networks to Prevent Ticket Replay Attacks", IACSIT International Journal of Engineering and Technology, Vol. 4, No. 3, June 